\title{
Protective Effects of Angiotensin-Converting-Enzyme-2 on Renal Dysfunction in Obstructive Jaundice
}

\author{
Mo Chen , Hongqian Wang , Erliang Kong, Jinmin Zhang, Weifeng Yu , Feixiang Wu * \\ Department of Anesthesiology \& Intensive Care, Shanghai Eastern Hepatobiliary Surgery Hospital, Second Military Medical University, Shanghai 200438 , P.R. \\ China
}

\section{Article Info}

\section{Article Notes}

Received: July 25, 2018

Accepted: September 18, 2018

\section{*Correspondence:}

Prof. Feixiang Wu, Department of Anesthesia \& Intensive Care, Shanghai Eastern Hepatobiliary Surgery Hospital, Second Military Medical University, 225 Changhai Road, Shanghai 200438, P.R. China;

E-mail: feixiangwu@hotmail.com.

( $2018 \mathrm{Wu} \mathrm{F}$. This article is distributed under the terms of the Creative Commons Attribution 4.0 International License.

\section{Key words:}

Renin-angiotensin-aldosterone system (RAAS)

Angiotensin-converting-enzyme-2 (ACE2)

Obstructive jaundice

Renal dysfunction

Renal protection

Aldosterone
Abstract

Acute renal failure occurring in patients with obstructive jaundice after surgery is still a serious clinical complication. Renin-angiotensin-aldosterone system (RAAS) plays a key role in the progression of kidney disease. Previous studies have demonstrated that angiotensin-converting-enzyme-2 (ACE2), a component of the RAAS system, acts as a local regulator for renal protection, and has a beneficial effect on renal fibrosis. This review will summarize the role of ACE2 and the protective effects on renal dysfunction in obstructive jaundice.

\section{Introduction}

Obstructive jaundice is a common clinical manifestation in hepatobiliary surgery. The pathophysiological changes in obstructive jaundice remain to be complex. Hyperbilirubinemia in patients with obstructive jaundice induces various clinical complications and increased morbidity and mortality including acute renal failure and endotoxemia ${ }^{1,2}$.

Acute renal failure occurs in 8 to $10 \%$ of patients with obstructive jaundice. However, the mortality rate of this complication is reaching up to $70 \%-80 \%{ }^{1}$. Accumulating evidences support that the increase of total bilirubin (Tbil) and serum creatinine (Scr) level in postoperative patients with obstructive jaundice relates to acute renal failure ${ }^{3,4}$.

A correlation was suggested between endotoxins and the complications in patients with obstructive jaundice ${ }^{1,5}$. Released cytokines due to endotoxin cause renal vasoconstrictive effect, acute tubular necrosis, fibrin deposition and systemic inflammatory response, which impact the short-term outcome ${ }^{6}$. Impaired immune function caused by obstructive jaundice contributes to the release of inflammatory cytokine TNF- $\alpha$, IL-1, IL- $6^{7}$.

The experimental animal model for obstructive jaundice is established by bile duct ligation (BDL) ${ }^{8}$. It has contributed to a better understanding of pathophysiology and the assessment of therapeutic strategy.

This review will explore the protective effects of RAAS (Reninangiotensin-aldosterone system) on renal dysfunction in obstructive jaundice.

\section{The RAAS and Renin Inhibition}

The RAAS has a crucial role in the regulation of blood pressure, fluid balance, and renal homeostasis 9 . Accumulating evidences have 
shown that the progression of renal disease is associated with RAAS ${ }^{10,11}$. Independent regulation of the intrarenal RAAS and inappropriate activation of this system contributes to the development and maintenance of renal disease ${ }^{12}$. Blockade of the RAAS by renin inhibition is an effective way to prevent progressive renal dysfunction, manifesting as reduced blood pressure, kidney fibrosis and inflammation, but at high risk of renal disease $\mathrm{e}^{13,14}$.

\section{The Role of ACEI/ARBs}

Previous research mainly focused on the effect of angiotensin-converting enzyme inhibitor (ACEI) and angiotensin receptor blockers (ARBs) on renal function. ACEI prevents the conversion of Angiotensin I to Angiotensin II by inhibiting the angiotensin-converting enzyme and decreases aldosterone secretion. Mishina et al. reported that renal dysfunction was improved after benazepril (an ACEI) was administered, which showed a protective effect in preventing the progression of renal disease ${ }^{15}$. ARBs block the attachment of Angiotensin II to its receptor. Similar to ACEI, ARBs decreased blood pressure and albuminuria, produced renal protective effect ${ }^{16}$. And, ACEI and ARBs are all recommended for the treatment of diabetic nephropathy ${ }^{17}$.

\section{The Role of ACE2 and Aldosterone}

Angiotensin-converting enzyme 2 (ACE2) is an exopeptidase that catalyzes the conversion of angiotensin II to angiotensin-(1-7) and is expressed abundantly in the kidney. It was found to have an important regulatory role in RAAS and was demonstrated to be a therapeutic target in renal disease. Studies have demonstrated the interplay between ACE2 and the kidney under normal and pathological conditions and pointed out the crucial role of ACE2 plays in the modulation of renal injury ${ }^{18,19}$. Clarke et al. concluded that the upregulation of ACE2 results in a significant protective effect on renal function in both diabetic patients and animal models ${ }^{20}$. Liu et al. studied the mechanisms of renoprotective role of ACE2 and demonstrated that enhanced Ang II-mediated TGF- $\beta /$ Smad and NF- $\kappa$ B signaling may be the mechanisms by which loss of ACE2 enhances renal fibrosis and inflammation ${ }^{21}$.

Studies using type 2 diabetes models have shown that ACE2 expression increased at an early stage, and reduced in the kidney with diabetic nephropathy developing ${ }^{22}$. Similarly, the ACE2 expression is elevated in early and decreased in the late stage of diabetic nephropathy in type 1 diabetes models ${ }^{23}$. Aldosterone is a steroid hormone produced by zona glomerulosa of the adrenal cortex in the adrenal gland. It is part of the RAAS and is related with the development and progression of the cardiovascular and renal disease. It was proved that aldosterone in the circulation indirectly promotes the development of renal diseases by inducing inflammation, fibrosis, and necrosis ${ }^{24}$.
Mechanism studies of aldosterone-induced inflammation provided the rationale for an expanded therapeutic role for mineralocorticoid receptor antagonists and aldosterone synthase inhibitors ${ }^{25}$. Fukuda et al. suggested that aldosterone induces kidney injury via activation of NF$\kappa \mathrm{B}$ and mineralocorticoid receptor, and decreased ACE2 expression may play an important role in aldosteroneinduced kidney injury ${ }^{26}$. Animal experiments showed that aldosterone administration could reduce the expression of ACE2. Nevertheless, aldosterone antagonists could reverse the pathological changes ${ }^{27}$.

\section{Aldosterone Antagonist: Spironolactone}

Spironolactone, a non-selective aldosterone antagonist, is commonly used in clinical practice which interferes with RAAS. The administration of spironolactone could abolish the effect of aldosterone.

de Sousa et al. showed that the treatment with spironolactone appears to be effective in controlling proteinuria and with a protective effect on renal fibrosis ${ }^{28}$. A meta-analysis evaluated the benefits and potential adverse effects of spironolactone on renoprotective treatment in patients with diabetic nephropathy and concluded that spironolactone could be used to prevent or slow diabetic nephropathy progression by reducing proteinuria ${ }^{29}$. Agrawal et al. concluded that ACEI and ARBs have been shown to suppress RAAS ineffectively, and they supported the use of spironolactone for more comprehensive suppression of the RAAS, which improved mortality outcomes in patients with chronic kidney disease $\mathrm{e}^{30}$.

Some animal experiments related with spironolactone have been conducted. Zhou $\mathrm{H}$ et al. proved that spironolactone may prevent renal fibrosis by inhibiting the endothelial-mesenchymal transition in rats ${ }^{31}$. Jeewandara et al. showed that inhibition of aldosterone via spironolactone was able to retard both renal and cardiac disease progression in a rodent model of kidney disease ${ }^{32}$. Another study showed that spironolactone administration after mild ischemia may be a useful therapeutic strategy to prevent the detrimental effect on renal function ${ }^{33}$.

In our previous study, we found that down-regulation of the ACE2 expression in the kidney of the BDL group was significant. Interestingly, the ACE2 expression is negatively correlated with Scr/Tbil. Further investigation showed that spironolactone intervention could significantly improve the renal fibrosis induced by obstructive jaundice. We also discovered that the ACE2 expression of BDL group could be upregulated by spironolactone. It implied that spironolactone could induce feedback regulation of RAAS, thereby affecting the expression of ACE2 and improving the renal function ${ }^{8}$. Nevertheless, the mechanism that leads to the expression change of ACE2 in obstructive jaundice is unclear and needs further study. 


\section{Summary}

In conclusion, the change of ACE2 expression was correlated with the renal dysfunction in obstructive jaundice. Spironolactone not only improved the progression of renal fibrosis but also upregulated ACE2 expression in the kidney of obstructive jaundice.

\section{Conflict of Interests}

Authors declared no conflict of interests.

\section{Funding}

None.

\section{References}

1. Green J, Better OS. Systemic hypotension and renal failure in obstructive jaundice-mechanistic and therapeutic aspects. J Am Soc Nephrol JASN. 1995; 5: 1853-1871.

2. Rege RV. Adverse effects of biliary obstruction: implications for treatment of patients with obstructive jaundice. AJR Am J Roentgenol. 1995; 164: 287-293.

3. Uslu A, Cayci M, Nart A, et al. Renal failure in obstructive jaundice. Hepatogastroenterology. 2005; 52: 52-54.

4. Mansfield SD, Sen G, Oppong K, et al. Increase in serum bilirubin levels in obstructive jaundice secondary to pancreatic and periampullary malignancy - implications for timing of resectional surgery and use of biliary drainage. HPB. 2006; 8: 442-445.

5. Greve JW, Gouma DJ, Buurman WA. Complications in obstructive jaundice: role of endotoxins. Scand J Gastroenterol Suppl. 1992; 194: 8-12.

6. Uslu A, Taşli FA, Nart A, et al. Human kidney histopathology in acute obstructive jaundice: a prospective study. Eur J Gastroenterol Hepatol. 2010; 22: 1458-1465.

7. Parlesak A, Schaeckeler S, Moser L, et al. Conjugated primary bile salts reduce permeability of endotoxin through intestinal epithelial cells and synergize with phosphatidylcholine in suppression of inflammatory cytokine production. Crit Care Med. 2007; 35: 23672374.

8. Kong EL, Zhang JM, An N, et al. Spironolactone rescues renal dysfunction in obstructive jaundice rats by upregulating ACE2 expression. J Cell Commun Signal. Epub ahead of print June 7, 2018. DOI: $10.1007 / \mathrm{s} 12079-018-0466-2$.

9. Nishiyama A, Kim-Mitsuyama S. New approaches to blockade of the renin-angiotensin-aldosterone system: overview of regulation of the renin-angiotensin-aldosterone system. J Pharmacol Sci. 2010; 113: 289-291.

10. Kobori H, Ozawa Y, Suzaki Y, et al. Young Scholars Award Lecture: Intratubular Angiotensinogen in Hypertension and Kidney Diseases. Am J Hypertens. 2006; 19: 541-550.

11. Nishiyama A, Konishi Y, Ohashi N, et al. Urinary angiotensinogen reflects the activity of intrarenal renin-angiotensin system in patients with IgA nephropathy. Nephrol Dial Transplant Off Publ Eur Dial Transpl Assoc - Eur Ren Assoc. 2011; 26: 170-177.

12. Kobori H, Nangaku M, Navar LG, et al. The intrarenal renin-angiotensin system: from physiology to the pathobiology of hypertension and kidney disease. Pharmacol Rev. 2007; 59: 251-287.

13. Feldman DL, Jin L, Xuan H, et al. Effects of aliskiren on blood pressure, albuminuria, and (pro)renin receptor expression in diabetic TG(mRen-2)27 rats. Hypertens Dallas Tex 1979. 2008; 52: 130-136.
14. Kelly DJ, Zhang Y, Moe G, et al. Aliskiren, a novel renin inhibitor, is renoprotective in a model of advanced diabetic nephropathy in rats. Diabetologia. 2007; 50: 2398-2404.

15. Mishina M, Watanabe T. Development of hypertension and effects of benazepril hydrochloride in a canine remnant kidney model of chronic renal failure. J Vet Med Sci. 2008; 70: 455-460.

16. Barnett AH, Bain SC, Bouter P, et al. Angiotensin-receptor blockade versus converting-enzyme inhibition in type 2 diabetes and nephropathy. N Engl J Med. 2004; 351: 1952-1961.

17. Levin A, Stevens PE, Bilous RW, et al. Kidney disease: Improving global outcomes (KDIGO) CKD work group. KDIGO 2012 clinical practice guideline for the evaluation and management of chronic kidney disease. Kidney Int Suppl. 2013; 3: 1-150.

18. Donoghue M, Wakimoto H, Maguire CT, et al. Heart block, ventricular tachycardia, and sudden death in ACE2 transgenic mice with downregulated connexins. J Mol Cell Cardiol. 2003; 35: 1043-1053.

19. Ortiz-Melo DI, Gurley SB. Angiotensin converting enzyme 2 and the kidney. Curr Opin Nephrol Hypertens. 2016; 25: 59-66.

20. Clarke NE, Turner AJ. Angiotensin-Converting Enzyme 2: The First Decade. International Journal of Hypertension. Epub ahead of print. 2012. DOI: $10.1155 / 2012 / 307315$.

21. Liu Z, Huang XR, Chen HY, et al. Loss of angiotensin-converting enzyme 2 enhances TGF- $\beta$ /Smad-mediated renal fibrosis and NF- $\mathrm{KB}$-driven renal inflammation in a mouse model of obstructive nephropathy. Lab Investig J Tech Methods Pathol. 2012; 92: 650-661.

22. Oudit GY, Liu GC, Zhong J, et al. Human Recombinant ACE2 Reduces the Progression of Diabetic Nephropathy. Diabetes. 2010; 59: 529-538.

23. Roca-Ho H, Riera M, Palau V, et al. Characterization of ACE and ACE2 Expression within Different Organs of the NOD Mouse. Int J Mol Sci; 18. Epub ahead of print March. 5, 2017. DOI: 10.3390/ijms18030563.

24. Hostetter TH, Rosenberg ME, Ibrahim HN, et al. Aldosterone in renal disease. Curr Opin Nephrol Hypertens. 2001; 10: 105-110.

25. Gilbert KC, Brown NJ. Aldosterone and inflammation. Curr Opin Endocrinol Diabetes Obes. 2010; 17: 199-204.

26. Fukuda S, Horimai C, Harada $\mathrm{K}$, et al. Aldosterone-induced kidney injury is mediated by NFKB activation. Clin Exp Nephrol. 2011; 15: 41-49.

27. Yamamuro M, Yoshimura M, Nakayama M, et al. Aldosterone, but not angiotensin II, reduces angiotensin converting enzyme 2 gene expression levels in cultured neonatal rat cardiomyocytes. Circ J Off J Jpn Circ Soc. 2008; 72: 1346-1350.

28. de Sousa MV, Guida JP, do Valle CF, et al. Spironolactone in PostTransplant Proteinuria: A Safe Alternative Therapy. Transplant Proc. 2017; 49: 813-816.

29. Hou J, Xiong W, Cao L, et al. Spironolactone Add-on for Preventing or Slowing the Progression of Diabetic Nephropathy: A Meta-analysis. Clin Ther. 2015; 37: 2086-2103.e10.

30. Agrawal S, Agrawal N, Garg J, et al. Heart failure and chronic kidney disease: should we use spironolactone. Am J Med Sci. 2015; 350: 147-151.

31. Zhou H, Xi D, Liu J, et al. Spirolactone provides protection from renal fibrosis by inhibiting the endothelial-mesenchymal transition in isoprenaline-induced heart failure in rats. Drug Des Devel Ther. 2016; 10: 1581-1588.

32. Jeewandara TM, Ameer OZ, Boyd R, et al. Protective cardiorenal effects of spironolactone in a rodent model of polycystic kidney disease. Clin Exp Pharmacol Physiol. 2015; 42: 353-360.

33. Barrera-Chimal J, Pérez-Villalva R, Ortega JA, et al. Mild ischemic injury leads to long-term alterations in the kidney: amelioration by spironolactone administration. Int J Biol Sci. 2015; 11: 892-900. 